\title{
Index rerum vol. 14
}

Page numbers of quotations from literature consisting of several pages are printed in italics Acetone Ill

Aerobacter, Cephaloridine 24 -, sulphonamides 276, 277 ALGOL 60275

Allyl-diethyl-chlorouracil 129, 158 Allyl-ethyl-propyl-chlorouracil 160 Allyl-ethyl-uracil 160

Allyl-methyl-nitrouracil 160 Allyl-methyl-propyl-chlorouracil 160 Allyl-methyl-propyl-uracil 160 Amantadine-HCl 141 Aminopenicillanic acid 115 Amphotericine 326, 328, 329 Ampicillin 115

Angiostrongylus cantonensis 244 Antiviral compounds 129,140,158 A-PR8 - Influenza virus 86 Biphenyl-ketaldehyde derivates 86 Bordetella pertussis, sulphonamides

153 -, trimethoprim 153 Busulfan 133

Carbenicillin, combination antibiotics 371

-, Pseudomonas 312

Cephaloridine 9, 22

Chest infections, chronic 151

Chlorambucil 133

Chloramphenicol 9, 234, 326, 328, 329

Chloroform Ill

Citrobacter, sulphonamides 153

-, trimethoprim 153

Clostridium, sulphonamides 153

-, trimethoprim 153

-, virginiamycin 153

Colistine, Pseudomonas 312

Combination of drugs 41,151, 356

Corynebacterium, virginiamycin 324

Cyclophosphamide 133

Cytosine arabinoside 133 Cytoxan 133

Diplococcus, sulphormethoxine 200

Dosage regimen calculation 273

Doxycycline 303

-, antibacterial activity 54,80

-, pharmacokinetic properties 176

-, respiratory tract infections 54

-, skin infections 101

-, urinary tract infections 77

drug action, kinetics 337

drug resistance 232

Dunning leukemia 65

Ehrlich carcinoma 65 
Endoxan 133

Enterobacter, sulphonamides 153

-, trimethoprim 153

Enterococci, sulphormethoxine 200

Enzymic proteins 234

Erythremia 133

Erythromycin 326, 328, 329

-, staphylococci 9

Escherichia coli 233

-, cephaloridine 24

-, doxycycline 81, 82,101

-, gentamicin 366,367

-, Lincomycin 337

-, sulphamethoxazole 153

-, sulphonamides 276, 277

-, sulphormethoxine 200

-, tetracyclines 253

-, trimethoprim 153

-, virginiamycin 324

Ethambutol 356

Ethyldeoxyuridine 129

Fanasil v. sulphormethoxine Flexner-Joblyng tumor 65 Fluoro-uracil 130 Friend-vi $\gamma$ us 158

Fucidine, staphylococci 9

392

Index rerum

Fusidic acid 113, 326, 328, 329 Gentamicin, antibacterial activity

366, 367 -, combination antibiotics 371 -, pediatric infections 366 -, pharmacokinetic properties 367 -, pseudomonas 312 Glycerol formal 227

Haemophilus, doxycycline 58

-, penicillin 306

-, sulphamethoxazole 153

-, sulphormethoxine 200

-, trimethoprim 153

-, virginiamycin 324

Herella vaginicola 366,367

Herpes virus 158

Hetacillin, clinical report 115

-, pharmacokinetic properties 118

Ibenzmethyzin 133 Isoniazid 358 -, detoxification 227 Kanamycin 234, 326, 328, 329 -,

staphylococci 9 Kelfizina v. Sulfalene Klebsiella, cephaloridine 24 -, doxycycline 58, 81, 82 -, gentamicin 366, 367 -, sulphamethoxazole 153 -, sulphormethoxine 200 -, trimethoprim 153

Leukemia L 121065 Leukeran 133 Lincomycin, E.coli 337 -, staphylococci 9 Listeria, virginiamycin 324 LPC-1 plasma cell tumor 65 Lysozyme 9

Madribon v. sulphadimethoxine Mammary adenocarcinoma E 0771 
65 Mast cell tumor P 81565 Methylcholanthrene 65 Methyl-isobutyl ketone Ill Murphy-Sturm tumor 65

Mutation 94

Mycobacteria, ethambutol 356

-, isoniazid 356

-, rifampicin 93, 356

-, streptomycin 356

-, virginiamycin 324

Mycoplasma cells 258

-, virginiamycin 324

Myleran 133

Natulan 133

Neisseria, sulphamethoxazole 153

-, sulphormethoxine 200

-, trimethoprim 153

-, virginiamycin 324

Neomycin 326, 328, 329

-, staphylococci 9

Nitrofurantoin, staphylococci 9

Novobiocin 113, 326, 328, 329

-, staphylococci 9

Oleandomycin, staphylococci 9

Pancreatine Ill

Paranasal sinus, cone, penicillin 303

Paromomycin, staphylococci 9

Pasteurella, sulphamethoxazole 153

-, trimethoprim 153

-, virginiamycin 324

Penicillin 234, 326, 328, 329

-, antibacterial activity 306

-, pharmacokinetic properties 303

-, protein binding 113

-, staphylococci 9

Perturbation 232

Ph-value, elimination 1

Plasma binding, virginiamycin 109

Pneumococci, cephaloridine 24

-, penicillin 306

Polycythemia rubra v. erythremia

Polymyxin, pseudomonas 312

Pristinamycin, staphylococci 9

Procarbazine 133

-, hydrochloride 65

Proteus, cephaloridine 24

-, doxycycline 58, 81, 82

-, gentamicin 366, 367 
Index rerum

393

-, rif ampicin 93

-, sulphamethoxazole 153

-, sulphonamides 276, 277

-, sulphormethoxine 200

-, trimethoprim 153

-, virginiamycin 324

Pseudomonas 81, 82

-, carbenicillin 312

-, cephaloridine 24

-, colistine 312

-, doxycycline 58

-, gentamicin 312, 366, 367

-, polymyxin 312

-, rifampicin 93

-, sulphamethoxazole 153

-, sulphonamides 276, 277

-, sulphormethoxine 200

-, trimethoprim 153

-, virginiamycin 322

Receptor destroying enzyme 86, 141 Resistance, development 93 Rifampicin, antibacterial activity 93,

356 Rifamycin staphylococci 9

Salmonella, cephaloridine 24

-, rifampicin 93

-, sulphamethoxazole 153

-, sulphormethoxine 200

-, tetracyclines 255

-, trimethoprim 153

Sarcina lutea, virginiamycin 324

Sarcoma 18065

Shigella, sulphonamides 153,200 ,

276, 277 -, trimethoprim 153 -, virginiamycin 324 Skin infections, doxycycline 101 Soframycin, staphylococci 9 Spiramycin, staphylococci 9 Staphylococci 14, 233 -, antibiotics 7 -,

cephaloridine 24 -, doxycycline 58,101 -, rifampicin 93 -, sulphonamides 153, 200, 276, 277

-, trimethoprim 153

-, virginiamycin 324

Staphylokinase 7, 14

Streptococci 81, 82

-, cephaloridine 24

-, doxycycline 58,102

-, penicillin 306

-, rifampicin 93

-, sulphonamides 153, 200, 276, 277 
-, trimethoprim 153

-, virginiamycin 324

Streptomycin 326, 328, 329, 357

-, staphylococci 9

-, sulphate 234

Sulfalene 37

Sulfisomidine 7

Sulphadimethoxine 37

Sulphamethoxazole 151, 275

Sulphamethoxypyrazine 273

Sulphonamides, antibacterial activity

276 -, dosage 273 -, formulas 196

-, pharmacokinetic properties 1, 273 Sulphormethoxine 195 Sulphorthomidine 37

Tetracycline 326, 328, 329

-, resistance 253

-, staphylococci 9

Tetramisole 244

Tinactin v. Tolnaftate

Tolnaftate 170

Trichophyton mentagrophytes 170

Trimethoprim, antibacterial activity

151 -, antimalarial activity 37 -, formula 37 -, pharmacology 38 Tyrothricin, staphylococci 9 Uterus epithelioma T 865

Vaccinia virus 158 Vibrio cholerae, sulphonamides 153 -, trimethoprim 153 Virginiamycin, antibacterial activity 322

394 Index rerum

-, pharmacokinetic properties 109, Walker carcinosarcoma 65

330

Xanthocillin, staphylococci 9

-, toxicity330 Xenalamine 140

Vxrulence 96 _ mechanism of action 86

WR 4629 v. Sulfalene Xenaldial 140 